\title{
Major Influencing Factors of the Mood of Physical Education Examinees in Yunnan Province
}

\author{
Yong Yang ${ }^{1, *}$ Xiaokun $\mathrm{Li}^{2}$
}

\author{
${ }^{1}$ Yunnan Normal University, Kunming, Yunnan 650500, China \\ ${ }^{2}$ Dongfang College, Shandong University of Finance and Economics, Tai'an, Shandong 271000, China \\ *Corresponding author.
}

\begin{abstract}
This paper investigated the main influencing factors on mood of examinees for college entrance examination in PE (physical education) in Yunnan province through questionnaire investigation, interview and literature. The results showed that the five factors that affect the mood of PE examinees in Yunnan province could be summarized as follows: social support, test anxiety, self-confidence, external interference and the test environment. The levels of the examinees' mood of different gender are mostly influenced by self-confidence. What's more, the examinees that had a long training period more cared about the test environment. Besides, uneven economic and education developments of Yunnan made the influencing factors on examinees' mood different greatly.
\end{abstract}

\section{Keywords: PE (physical education) examinee, mood, influencing factor}

\section{INTRODUCTION}

Under the background that the score of college entrance examination is the only criterion for examinees to enter colleges and universities, many examinees and parents regard it as a fateful moment. The pressure of going to school, parents' expectation and society's expectation all make examinees bear heavy psychological burden. As an important link in the selection of sports talents, the college entrance examination for sports affects the hearts of sports workers at all levels. Comparing with professional athletes, sports students have their own particularity; they generally have shorter training years, lower level of competition, competition experience and other problems. In addition, they have to learn the knowledge of culture and take part in daily physical training. All these things make them easy to be disturbed before the exam. In modern scientific and transparent training, the influence of psychological factors on individual performance is becoming more and more obvious. In the physical education college entrance examination, there are also many examples to result in failure because of nervousness repeatedly .for example, basketball candidates often miss the shooting; track and field candidates have many times false start ; martial arts candidates bring about panic movement deformation.

For examinees in high school, the characteristics of psychological development in this period determine that their emotions are more complex with literary decorations and implicit nature. Therefore, their emotions are more represented by the state of mind[1] State of mind is a relatively persistent and weak emotional state that infects all emotional experiences with a certain color, which has the characteristics of stability and dispersion. It will affect the subject's attitude to things in a period of time, and then affect people's behavior. As the old saying goes, when different people face the same thing, "those who are worried are worried, while those who are happy are happy when they see it". It shows that people with different states of mind have different attitudes towards problems. For examinees, a positive and optimistic state of mind will help them improve the efficiency of activities, enhance confidence, and give full play to their potential. However, a negative state of mind may make them upset and emotionally disturbed [2].

Through literature review, it is found that there are more studies on the pre-competition psychology of professional athletes, but less attention paid to the group of sports examinees. In some existing psychological surveys of sports examinees, the research means are mostly to measure the mental health level of examinees before the examination through a single scale, and to infer the psychological influencing factors of examinees according to the scores of each latitude. In the search for the source of the problem, "may", "perhaps", "probably" and other words are more used, which also makes the proposed recommendations with too much subjective color, reducing the scientific nature and accuracy of the research. This paper attempts to explore the causes of students' psychological problems and make clear the direction of guidance through empirical investigation on the influencing factors of 
students' mood in Yunnan province. It will provide reference and basis for the psychological research of PE examinee in the future.

\section{METHOD}

\section{A. Data sample}

The research respondents of this paper were 947 examinees who took the physical education examination for college enrollment in Yunnan province in 2019. Of these 947 examinees, 647 of them were male, and 300 of them were female. Sixty-six percent of the respondents were ethnic Han, and others were minority. The training experience of the respondents included 1 year $(n=357), 2$ year $(n=395)$ and 3 year $(\mathrm{n}=178)$. The respondents were distributed among 16 cities in Yunnan province.

\section{B. Measures}

1) Collection of survey items: By visiting sports examinees and coaches and summarizing the psychological research experience of sports examinees based on the literature, this paper made a preliminary arrangement of the influencing factors of examinees' mood before the examination. The pre-selected influencing factors were submitted to the experts in the field of sports psychology for their judgment. By defining the five grades of very important, important, uncertain, unimportant and very unimportant and using the comprehensive attitude method to intercept factors with the coefficient scoring standard $(\mathrm{F}>0.5)$, the original questionnaire with 28 items was preliminarily obtained.

2) Item analysis: As the immediate environment of the college entrance examination could not be copied and simulated, the questionnaire on influencing factors of college students was not suitable for repeated tests. We further examined and sorted out the questionnaire of the influencing factors through the operation of discrimination and factor analysis.

- Differentiation method: first, score the reverse items of the influencing factor scale. According to the total score of the scale, $27 \%$ was defined as high group, and $27 \%$ was defined as low group. In order to test the difference between the high and low group, the $\mathrm{T}$ test was used to find that the test results of 28 questions all reached a significant level $(\mathrm{P}<0.01)$, therefore, we could think that all 28 questions could identify the response degree of different students [3]

- Factor analysis method: the factor analysis method was used to investigate the load of each item on factors. Items less than 0.35 for any factor were deleted. As a result, 3 items were deleted. Finally, the questionnaire was composed of 25 items.

\section{RELIABILITY AND VALIDITY}

\section{A. Reliability test}

The 5-factor model was obtained by factor analysis of the questionnaire. This study was not suitable for the external reliability test of the retest. So we used the kclonbach coefficient to test its internal consistency. After the test, the reliability coefficient of 5 factors was between 0.629 and 0.832 , and the overall score coefficient of the scale was 0.832 , indicating that the reliability of the questionnaire was satisfactory.

\section{B. Validity test}

This questionnaire was formed through actual survey and interview, which was combined with a large number of literatures and screened and revised by experts, its content validity was acceptable. Structural validity: the principal component analysis method was used to test the validity of the concept, and it was found that $84 \%$ of the item commonality was more than 0.5 . Since item commonality was a sign of the scale's structural validity, the conceptual validity of this questionnaire was acceptable.

\section{RESULT AND ANALYSIS}

\section{A. Factor analysis on the influencing factors}

First, KMO value test were performed on the 25 items after elimination. The results showed that the KMO value was 0.872 , the spherical value was 5256.267 , and the degree of freedom was 300 , reaching a significant level. In order to explore the influencing factors of physical education examinees' mood, we conducted factor analysis on the influencing factors questionnaire to intercept the factors with the characteristic root greater than 1 . The Results showed that 25 items were explained by 5 factors, and the cumulative variance contribution rate was $56.894 \%$. 
TABLE I. FACTOR ROTATION MATRICES

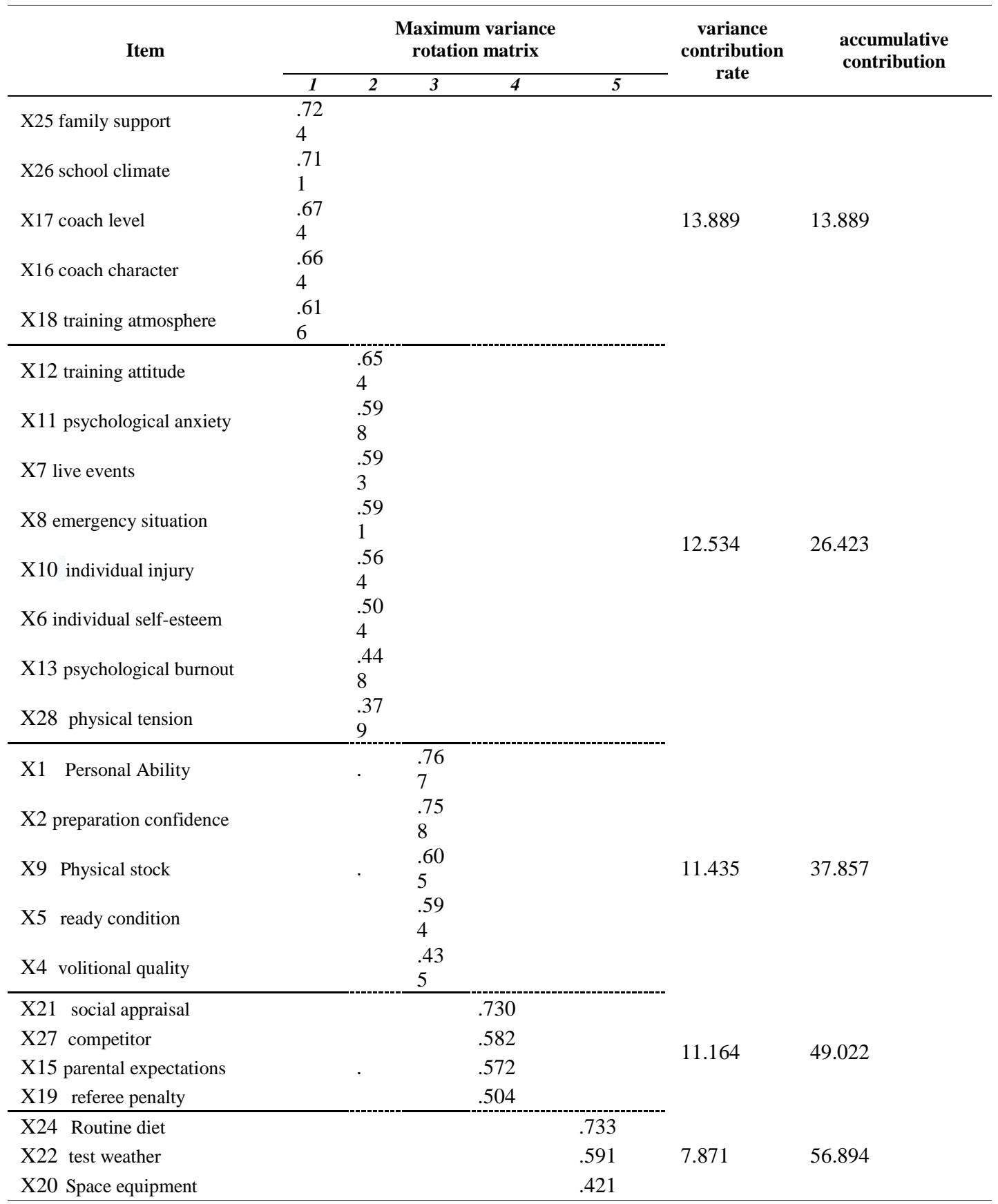

${ }^{\text {a. }}$ Note: Intercept factor with load of 0.35

By means of the maximum variance method, the factor matrix was rotated and sorted according to the load of each item in the matrix, and the 25 items were clearly marked into each factor (see "Table I"). Among them, family support, school environment, coach's level and personality, training atmosphere and other items fit together. Family, school, coach and training environment basically covered the social scope that examinees could contact, they need social support and expectation on them. So we could call factor 1 social support. They worried about training preparation, life trifles and injuries, competition emergencies and failure, physical tension and psychological preparation fatigue, which all came from the stress response to the college entrance examination. According to the general explanation of psychology, we named factor 2 as test 
preparation anxiety. Factor 3 contained confidence and affirmation in one's own athletic ability and physical reserve, and the will quality of daring to deal with difficulties. So, we named factor 3 self-confidence. Factor 4 reflected that sports examinees cared about the referee's decision, external evaluation, and the performance of the opponent. We named this factor external interference, and it was worth mentioning that external interference mainly came from external pressure experienced by examinees after they came to the examination environment, which was different from social support factors. Factor 5 was composed of the discomfort of site equipment, weather, rest and diet, which could be regarded as the environmental factor of the examination. By analyzing the variance contribution rate of 5 factors, we ranked them in order of importance: social support, preparation anxiety, selfconfidence, external interference and examination environment.

The living and learning environment of physical education candidates was between home and school, and the advice and support of family, coach and school often played an important role in guiding students' learning and training. Psychological social support was generally subjective and objective. For high school students, Objective social support including material direct assistance and social networking was less obvious. However, subjective emotional experience was very important among students, and whether students' emotions were supported, recognized and understood would determine the level of individual psychological adjustment [4]. Only when their emotions and opinions were fully supported by the society could they inspire positive emotions and selfconfidence [5]. Good school environment and training atmosphere were conducive to their learning and training activities, family support kept them safe, and full trust in the coaching level of the coach provided a strong guarantee. But social support might be a doubleedged sword. Too much social concern could also become a psychological burden for some examinees. Especially some coaches and parents put forward high expectations and requirements for their children, which made students bear heavy pressure and easily form a disordered state of mind. As the support of the school, parents and coaches for children, it was more important to put energy into communication and guidance, paid attention to their pressure reduction and self-esteem maintenance before the exam, and did not put too much expectation into the students.

Examinee preparation anxiety was mostly caused by the stress source of the college entrance examination. Due to the particularity and importance of the college entrance examination, it was easy for examinees to perceive the examination situation as the potential danger of the individual reality, which would produce the initial physiological reaction. For example, they would fell muscle tension, cold sweats, rapid heart rate, frequent urination, etc. Due to the differences in school training conditions, teaching levels and individual training attitudes, examinees often showed concerns about their daily training and anxiety about exam preparation when facing with strong test stimuli [6]. This was especially obvious for candidates with poor physical fitness, passive test preparation and weak nerve types. How to let the examinee dare to face the test challenge, adjust the physical and psychological panic was the key problem to solve the test preparation anxiety. The examinee need to adjust attitude, down-toearth, perseverance in the usual training. Therefore, Coaches and parents should analyze and deal with the sources of examinee anxiety. Physiological adverse reactions should be timely found and effectively solved for the examinee before the examination.

Vealey(1986), a foreign scholar, once made such a definition of sports confidence: "the belief or degree of conviction of an individual's ability to succeed in competitive sports" [7]. In other words, this kind of firm belief of the examinee was higher, the selfconfidence level was higher, which was advantageous to the movement performance. The examinee's affirmation to the athletic ability and the physical strength reserve showed the full faith in the individual ability. When the exam was approaching, candidates with strong confidence were in high spirits and nervous excitement, believing that they could overcome the special circumstances in the exam. On the contrary, if the examinee was negative to prepare for the test, their self-confidence was insufficient, which would be timid and restrict their performance. As for how to adjust the pre-test confidence of candidates, some researchers believed that athletes with strong motivation generally had a good grasp of their own ability and were very confident. Therefore, in order to improve the examinee motivation, it was very important to set up a good goal and strengthen the encouragement of education.

When examinees came to a strange competition environment, psychology was easy to have a contrast. Especially for the lower level of sports examinees, facing with the same project examinees of high performance, they were easier to lose self-confidence. This required us to do such a job of encouraging students' confidence that they do not fear the challenge of opponents. High school was a sensitive period for students' self-emotional development. We must pay attention to the influence of our words and deeds on students. Could candidates from different regions adapt to the environment and climate after coming to the test area? Could they eat and live in the surrounding area well and could they adapt to the equipment and venues? All these reflected the preparation of the training team. The discomfort of the environment also produced a bad state of mind. For example, if there was a rain during the college entrance examination, many coach and 
candidates would complain to affect their performance to some extent. In addition, through the interview with the coach and examinee, we found that training conditions of many training team were poor, they had a sense of discomfort for the new site. Therefore, it was essential to adapt to the site, climate and diet for coaches in advance.

\section{B. Variance analysis on the influencing factors}

1) Analysis between different genders: Some scholars mentioned in the study on the pressure of teenagers that girls tend to have a stronger sense of the pressure to enter college than boys, and they more concerned about the success or failure of competitions. Through the t-test, there was a significant difference in confidence factor scores between male and female students $(\mathrm{T}=2.287, \mathrm{P}<0.05)$. Girls had lower confidence scores than boys. This was also mentioned in Zhu jianmin's psychological research on female athletes [8]. Further observation showed that girls scored higher than boys in social support, test preparation anxiety, external interference and test environment factors, indicating that they were more emotionally sensitive. Therefore, coaches should pay more attention to the changes of girls' mood in the training team and maintain their self-esteem and selfconfidence.

2) Analysis among different training time: Through the actual investigation of the candidates for the sports college entrance examination, we found that the time for the candidates to participate in the training was concentrated in the high school stage. We divided the training time into three stages, which were 1 year (including less than 1 year), 2 years, 3 years and more. MANOVA analysis was conducted on the difference of mood influencing factors among candidates with different training years. It was found that there were significant differences in the scores of examinee factors with different training years (Lambda of Wilks $=0.976$, $\mathrm{F}=1971, \mathrm{P}<0.05)$, the examination environment was the main factor of the differences. After multiple comparisons, candidates with a training time of 3 years or more had higher scores than those of 1 year or less and 2 years $(\mathrm{P}<0.01)$. This may be that candidates with longer training time tend to be more sensitive to the environment of the examination because of the accumulation of training experience and stable performance. Therefore, for examinees with a long training time, coaches should reduce their psychology of "details determine success or failure" before the test, allowing them to focus more on the completion of the action of the competition instead of worrying about the surrounding environment, which would help them concentrate and play their due level[9].

3) Analysis between different regions: Through $\mathrm{T}$ test of the city and rural examinees mood influence factor score, the results showed that there were significant differences in 5-factor scores between urban and rural areas $(\mathrm{P}<0.05)$. This showed that the level of economic education had a great influence on the psychological causes of examinees. Further observation showed that the social support and examination environment factors of rural examinees were higher, which was mainly because of less developed economic education for students in regions. Therefore, they hoped to change their fate through the college entrance examination and paid more attention to social expectations. The lack of investment in education made some areas have poor training conditions, and the training and examination cannot be well integrated, which also made them pay more attention to the influence of equipment and venues. City students scored high on test preparation anxiety, external interference and low on confidence factor. This was mostly due to the fact that students in economically developed areas were exposed to more external things and had higher cognitive anxiety.

\section{CONCLUSION}

The conclusion and advice are as follows:

- The influencing factors of the mood of Yunnan physical education candidates could be summarized as social support, preparation anxiety, self-confidence, external interference and examination room environment.

- Girls scored lower in confidence factor and Candidates with longer training years scored higher on environmental factors. The examinees in underdeveloped areas cared about social expectations, examination environment factors. The examinees in developed regions were susceptible to preparation anxiety and external interference.

- Generally speaking, the main factor that affected the mood level of examinees was the psychological burden. In order to reduce the pressure of candidates' expectations, the primary problem to solve is to set up a reasonable goal orientation.

- Before the test, candidates were prone to show panic and anxiety about the environment, which required us to check the source of anxiety according to individual conditions. It was very important to encourage their confidence and 
make them adapt to the competition environment.

- Coaches should try their best to improve the girls' confidence in the training team and reduce the anxiety of students who have been in the training for a long time. At the same time, the relevant departments should supply better training conditions for underdeveloped areas.

\section{References}

[1] Ye Yi-Gan, Zhu Bei-Li.Psychology (Revised) [M]. Shanghai: East China Normal University Press, 1996.

[2] Ji Liu. Sports Psychology [M]. Beijing: Higher Education Press, 2010.128.

[3] Wei Jinshui, Zou Liangchou, Pan Huiwen.Quantitative Analysis of Internal Reliability And Structural Validity of Self-compiled Scales in Sports Research [J]. Journal of Guangzhou Institute of Physical Education, 2006,25 (6) : 110-114.

[4] Zhang Xiangkui, Zhang Lin, Ma Liwen.A Study on the Relationship Between Cognitive Evaluation, Sense Of Psychological Control, Social Support And College Entrance Examination Stress [J]. Psychological Development And Education, 2003,18 (3) : 74-79.

[5] Liang Jianping, Chang Jindong. Comparative Analysis of Competition Confidence Sources of Elite Athletes In China Taking Athletics And Shooting As Examples [J]. Sports Science, 2007,27 (9) : 41-42.

[6] Yao Anquan, Zhang Jianfeng, Wang Zhiluo. Causes and Coping Strategies of Sports Technology Test Anxiety of Sports College Students [J]. Shaolin and Tai Chi, 2011(22) : 47-50.

[7] Vealey R S. Conceptualization of Sport - Confidence And Competitive Orientation: Preliminary Investigation and Instrument Development [J]. Journal of Sport Psychology, 1986.

[8] Zhu Jianmin, Pan Guoping, Wang Shunxia. Research On Athletes' Anxiety Before Competition [J]. Anhui Sports Science and Technology, 2018(4).34-38.

[9] Fu Bin, Luo Mingdong. New Ideas And Practices In Contemporary Education [M]. Chengdu: Sichuan University Press, 2016. 